\title{
Continuous Reduced Graphene Oxide Film Prepared by Stitching of Nanosheets at the Interface of Two Immiscible Solutions
}

\author{
Youngku Sohn, ${ }^{*}$ Seog K. Kim, and Bong-Ki Min ${ }^{\dagger}$
}

\author{
Department of Chemistry, Yeungnam University, Gyeongsan, Gyeongbuk 712-749, Korea. *E-mail: youngkusohn@ynu.ac.kr \\ $\dagger$ Instrumental Analysis Center, Yeungnam University, Gyeongsan 712-749, Korea \\ Received April 20, 2010, Accepted November 29, 2010
}

Key Words: Reduced graphene oxide, Electron microscopy, UV-Vis absorption, Raman, XPS

\begin{abstract}
Graphene, an atom-thick two-dimensional (2D) honeycomb lattice sheet of $\mathrm{sp}^{2}$-bonded carbon atoms, has recently been emerged as a new promising material in various fields. ${ }^{1-4} \mathrm{Be}-$ cause of its gigantic charge carrier mobility it could be applied to field-effect transistors as a substitute of silicon. ${ }^{5,6}$ Due to its transparency and high electrical conductivity, it could be used as a substitute of ITO electrode in solar cells and light-emitting diodes. ${ }^{7,8}$ Other superior properties include large surface area, flexibility, strength, stiffness, and thermal conductivity. These provides wide applications of graphene including supercapacitor, battery, sensor, storage and drug delivery. ${ }^{1-4,9,10}$ For real applications, large-scale of graphene sheets or films needs to be prepared. Large-area (orders of centimeters) graphene films have recently been fabricated using a chemical vapor deposition (CVD) method on various metal substrates. ${ }^{4,11-12}$ This method requires a transfer process from the metal substrates to a desired substrate such as a transparent plastic sheet. Simpler methods are a thermal reduction of graphene oxide film prepared via the filtration of graphene sheets, Langmuir-Blodgett (LB) layerby-layer assembly, and filtration of graphene sheets followed by a film transfer. ${ }^{13-16}$ Investigators have now actively pursued various simpler ways of fabricating continuous graphene films or papers. Highly motivated by this, we have challenged to synthesize large-area/scale graphene films from solution.
\end{abstract}

\section{Experimental Section}

Graphene oxide (GO) was synthesized by a modified Hummers method. ${ }^{18,19}$ Graphene (or reduced graphene oxide, RGO) sheets dispersed in water were prepared by reduction of GO.

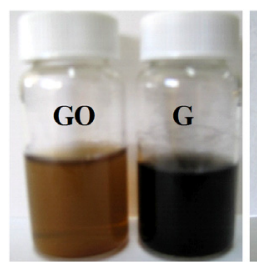

(A)

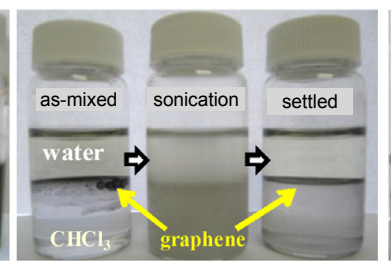

(B)

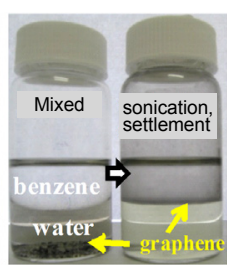

(C)
Figure 1. Photograph images (A) graphene oxide (GO) and RGO sheets dispersed in water solution. (B) RGO sheets in immiscible water and chloroform solutions before and after sonication, and after settlement of the sonicated solution. (C) graphene sheets in immiscible water and benzene solutions before sonication and after sonication followed by settlement.
For the reduction process, we added an appropriate amount of ascorbic acid into a GO-dispersed water solution, ${ }^{20}$ and stirred for more than two days to ensure complete reduction. As the GO reduces to graphene, the color changes from brown to black color (Figure 1A). The RGO solution was mixed with various immiscible solvents; chloroform $\left(\mathrm{CHCl}_{3}\right)$, toluene, benzene, chlorobenzene and hexane.

For Figure 1B, we mixed the RGO-dispersed water solution with chloroform, sonicated for at least $10 \mathrm{~min}$, and let the solution settle overnight. Before sonication, the RGO is dispersed in water and some agglomerates sit at the interface (see Supporting Information Figure S1). Upon sonication, the mixed solution turns milky white with a dull interface. The bottom solution becomes darker than the top upon sonication. After settlement of the milky solution, the top and bottom solutions become clear, and a continuous RGO-film fully forms at the sharper interface (see Supporting Information Figure S1). For a solution mixed with benzene and RGO-dispersed water (Figure 1C), upon sonication and settlement the RGO sheets and agglomerates resided in water side form a continuous film at the interface. It is clearly seen that the concave interface changes to a flat interface, an indication of forming a continuous film. The film was transferred to a Si substrate for SEM (Hitachi 4800), Raman (WITec Alpha300R Confocal Raman Microscope, $633 \mathrm{~nm}$ ) and XPS (Thermo-VG Scientific MultiLab 2000 with Al Ka X-ray source) measurements. For UV-vis absorption (Jasco V530) and TEM (Tecnai G2 F20 S-TWIN), the film was onto a quartz substrate, and a $\mathrm{Cu}$ grid, respectively.

\section{Results and Discussion}

The continuity and flexibility of the film can be easily checked and observed by naked eye. For Figure 2 (top), we put heavy chloroform solvent onto the RGO-film at benzene-water interface, and then the film wraps, stores, and not to allow the solvent to fall on the bottom of the vial. Upon adding more chloroform, the film stretches to store more solvent; we will call it a graphene pocket or bag (see Supporting Information Figure S2). The graphene pocket shrinks back upon withdrawing of the chloroform. Upon further pipetting the solvent, the pocket shrinks further to a smaller size. Wrinkles in the film are seen in Figure 2 (bottom). By tilting the vial, we pull the curly lines (wrinkles) straight. Fascinatingly, we also observed that the RGO-film exhibits selfhealing character by stretching the wrinkles if externally da- 


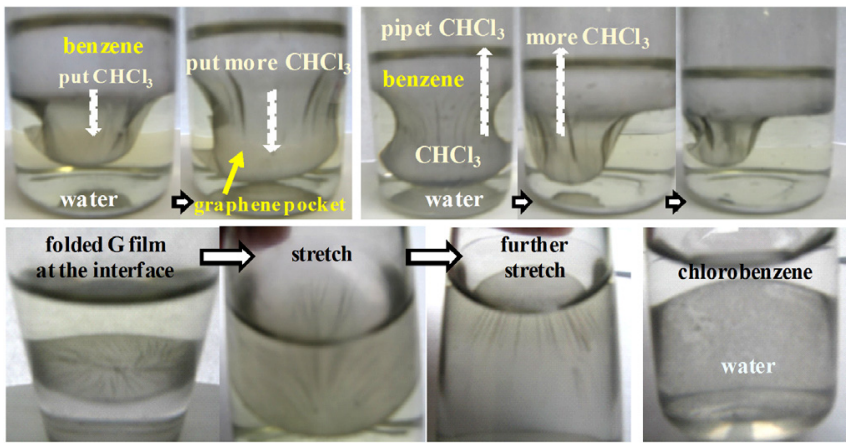

Figure 2. Photograph images Top: Expansion of graphene pocket upon adding chloroform, and contraction of the pocket upon withdrawing the solvent. Bottom left three: Stretching of folded graphene film. bottom right: discontinuous sub-millimeter size G-films floating on water with chlorobenzene on top.

maged. Although the film is severely damaged, a sonication for less than $60 \mathrm{sec}$ is enough to repair the film. This property could be applied to biotechnology. Among the solvents we have tried, chlorobenzene only did not show continuity (Figure 2, bottom right). The lateral dimension of the films is sub-millimeters in this solution. To check another polar solvent mixable with water, we replaced the top water solution with ethanol. Then, the continuous film at the interface becomes broken up into smaller size film (see Supporting Information Figure S3). However, the broken film becomes continuous when the ethanol is replaced by water back. During this replacement from ethanol to water we found very tiny bubbles forming at the interface. It appears that these bubbles assist the self-assembly of graphene sheets to form a continuous film.

A RGO-film on a quartz substrate $(2 \mathrm{~cm} \times 1 \mathrm{~cm})$ was prepared by a scooping method. We wrote YU (inset Figure 3 ) by a scratching method to ensure the RGO film. We measured the transmittance and absorbance of the film. An absorption peak is seen at $269 \mathrm{~nm}$, attributed to $\pi \rightarrow \pi^{*}$ transition of $\mathrm{C}=\mathrm{C}$ bonds. This is in good agreement with the literature. ${ }^{17}$ For a thinner film, the transmittance increases from $77 \%$ at $400 \mathrm{~nm}$ to $87 \%$ at $1100 \mathrm{~nm}$, corresponding to a film consisting of about seven

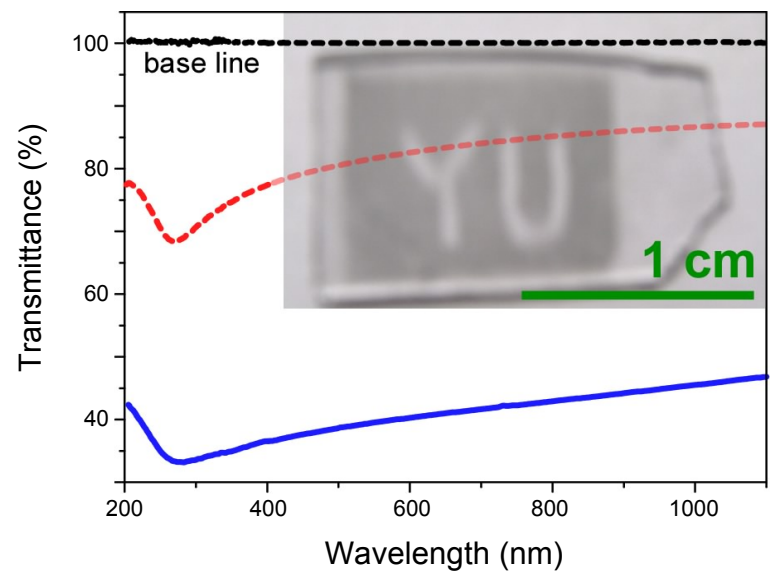

Figure 3. UV-vis. transmittance (absorbance) spectra of continuous (thin and thick) films (see inset photograph) transferred onto a quartz substrate.

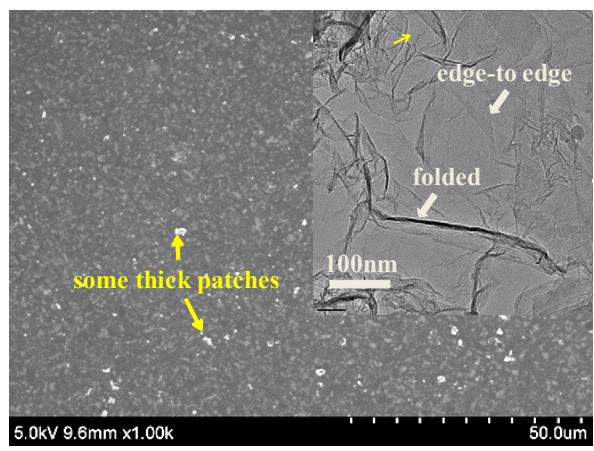

Figure 4. SEM and TEM (inset) images of RGO-film transferred onto a silicon substrate and a $\mathrm{Cu}$-grid, respectively.

graphene sheets. ${ }^{21}$ For the absorbance of a thicker film, although the absorbance is relatively more increased in the visible region the whole spectrum shows no critical change with film thickness.

Figure 4 shows the SEM and TEM images of the RGO-film. From the TEM image, it appears that graphene sheets are connected by edge-to-edge stacking or folding to form a continuous film. The size of each RGO sheet deduced from the TEM image is in sub-micron size. As seen in the SEM image, the RGO film is smooth and continuous, but some thick patches (or aggregated graphene sheets) are present. The number of patches could be minimized by replacing the top water with new fresh water solvent several times.

We have further characterized the RGO film using XPS and Raman. The XPS and Raman spectra in Figure 5 indicate the formation of RGO sheets, in good agreement with the literatures. $^{20,22-24}$ The $\mathrm{C} 1 \mathrm{~s}$ XPS spectrum can be resolved into four peaks at 284.3, 286.3, 287.5, and $288.9 \mathrm{eV}$, attributed to the carbons of $\mathrm{C}-\mathrm{C}, \mathrm{C}-\mathrm{O}, \mathrm{C}=\mathrm{O}$, and $\mathrm{O}-\mathrm{C}=\mathrm{O}$, respectively. Although the peak at $284.3 \mathrm{eV}$ is dominantly seen, the $\mathrm{C} 1 \mathrm{~s}$ XPS indicates that the RGO sheets still contain some oxygenated carbon species. The full reduction of GO has been a great challenging work. ${ }^{24}$ For the Raman spectrum, the $\mathrm{D}$ band at $1340 \mathrm{~cm}^{-1}$ is attributed to in-plane $\mathrm{sp}^{2}$ carbon $\mathrm{A}_{1 \mathrm{~g}}$ mode. This peak also depends on the defects in a graphene system. The corresponding two-dimensional (2D from $2 \times 1340 \mathrm{~cm}^{-1}$ ) peak by double resonance process is found at $2680 \mathrm{~cm}^{-1}$. The $\mathrm{G}$ band at 1584 $\mathrm{cm}^{-1}$ is attributed to the $\mathrm{E}_{2 \mathrm{~g}}$ mode of $\mathrm{sp}^{2}$ carbons, and known to be nearly absent for pristine graphite..$^{20,22-24}$ The S3 peak at around $2910 \mathrm{~cm}^{-1}$ is known to be due to the second-order of a combination of $\mathrm{D}$ and $\mathrm{G}$ peaks. ${ }^{25}$
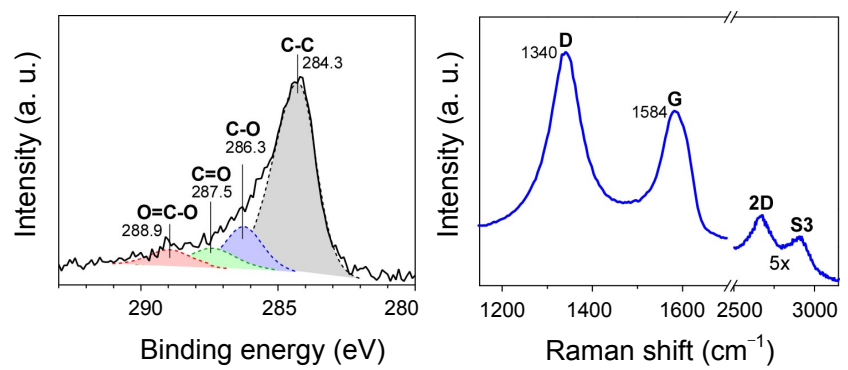

Figure 5. C 1s XPS (Left) and Raman (Right) spectra of a RGO film transferred on to a Si substrate. 


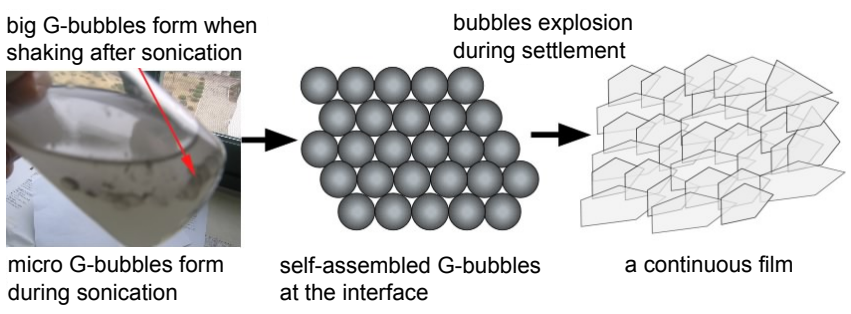

Figure 6. Schematics of forming a continuous RGO film at the immiscible solution-solution interface.

Figure 6 displays a plausible mechanism of forming the continuous RGO-film. We assume that the micron size bubbles of RGO sheets form by surface tension effects during sonication, quickly self-assemble at the interface, and then when the G-bubbles are broken the RGO sheets stack (or fold) edge-to-edge to form a continuous film, as displayed in Figure 6. Upon shaking the solution by hand after sonication, we observed that big graphene $(\mathrm{G})$-bubbles form, and break to form large sub-centimeter size graphene films. The formation of G-bubbles during sonication might be a key step of forming a continuous RGO-film.

\section{Summary}

In summary, RGO sheets dispersed in water are prepared by chemical reduction of $\mathrm{GO}$ using ascorbic acid. By mixing and sonication of submicron-size RGO sheets in two immiscible liquids (e.g., chloroform and water) for the first time we have prepared a continuous large-area RGO film at the interface. In other words, we have shown that aggregated RGO sheets could be fully stretched at the interface to form a continuous film. The RGO film has been characterized by SEM, TEM, UV-vis absorption, XPS and Raman. The film exhibits high flexibility, transparency, and very long-term stability without forming aggregations. Without requiring vapor deposition, a special instrument, or a filtration followed by a removing the filter paper one could easily achieve a continuous RGO-film in any laboratories. Our solution-based method is much simpler and cost-effective, and very good for large scale mass production. This finding could boost real applications of graphene in laboratory and industry, and provide a new methodology for the fabrication of large-area continuous graphene films.

Acknowledgments. This work was supported by the Na- tional Research Foundation of Korea (Grant no. 2009 0083855).

\section{References}

1. Allen, M. J.; Tung, V. C.; Kaner, R. B. Chem. Rev. 2010, 110, 132.

2. Choi, W.; Lahiri, I.; Seelaboyina, R.; Kang, Y. S. Crit. Rev. Solid States Mater. Sci. 2010, 35, 52.

3. Rao, C. N. R.; Sood, A. K.; Voggu, R.; Subrahmanyam, K. S. J. Phys. Chem. Lett. 2010, 1(2), 572.

4. Geim, A. K.; Novoselov, K. S. Nat. Mater. 2007, 6, 183.

5. Li, X.; Cai, W.; An, J.; Kim, S.; Nah, J.; Yang, D.; Piner, R. et al. Science 2009, 324, 1312.

6. Meric, I.; Han, M. Y.; Young, A. F.; Ozyilmaz, B.; Kim, P.; Shepard, K. L. Nature Nanotechol. 2008, 3, 654.

7. Wang, X.; Zhi, L. J.; Müllen, K. Nano Lett. 2008, 8, 323.

8. Wu, J.; Agrawai, M.; Becerril, H. A.; Bao, Z.; Liu, Z.; Chen, Y. Peumans, P. ACS Nano 2010, 4(1), 43.

9. Lu, G.; Ocola L. E.; Chen, J. Nanotech. 2009, 20, 445502.

10. Sun, X.; Liu, Z.; Welsher, K.; Robinson, J. T.; Goodwin, A.; Zaric, S.; Dai, H. Nano. Res. 2008, 1, 203.

11. Kim, K. S.; Zhao, Y.; Jang, H.; Lee, S. Y.; Kim, J. M.; Kim, K. S.; Ahn, J. H. et al., Nature 2009, 457, 706.

12. Reina, A.; Jia, X.; Ho, J.; Nezich, D.; Son, H.; Bulovic, V.; Dresselhaus, M. S.; Kong, J. Nano Lett. 2009, 9, 30.

13. Xu, Y.; Bai, H.; Lu, G.; Li, C.; Shi, G. J. Am. Chem. Soc. 2008 , 130,5856

14. Eda, G.; Fanchini, G.; Chhowalla, M. Nature Nanotechnol. 2008 , $3,270$.

15. Li, X.; Zhang, G.; Bai, X.; Sun, X.; Wang, X.; Wang, E.; Dai, D. Nature Nanotechnol. 2008, 3, 538.

16. Lee, J. H.; Shin, D. W.; Makotchenko, V. G.; Nazaro, A. S.; Fedorv, V. E.; Kim, Y. H.; Choi, J.-Y.; Kim, J. M.; Yoo, J.-B. Adv. Mater. 2009, 21,1 .

17. Li, D.; Muller, M. B.; Gilje, S.; Kaner, R. B.; Wallace, G. G. Nature Nanotechnol. 2008, 3, 101.

18. Park, S.; Ruoff, R. S. Nature Nanotechnol. 2009, 4, 217.

19. Cote, L. J.; Kim, F.; Huang, J. J. Am. Chem. Soc. 2009, 131, 1043.

20. Zhang, J.; Yang, H.; Shen, G.; Cheng, P.; Zhang, J. Guo, S. Chem. Commun. 2010, 46(7), 1112.

21. Nair, R. R.; Blake, P.; Grigorenko, A. N.; Novoselov, K. S.; Booth, T. J.; Stauber, T.; Peres, N. M. R.; Geim, A. K. Science 2008, 320, 1308.

22. Liu, Y.; Gao, L.; Sun, J.; Wang, Y.; Zhang, J. Nanotechnology 2009, 20,465605

23. Kim, N.; Oh, M. K.; Park, S.; Kim, S. K.; Hong, B. H. Bull. Korean Chem. Soc. 2010, 31, 999.

24. Moon, I. K.; Lee, J.; Ruoff, R. S.; Lee, H. Nature 2010, doi:10.1038/ ncomms 1067.

25. Wang, H.; Robinson, J. T.; Li, X.; Dai, H. J. Am. Chem. Soc. 2009, 131,9910 .

26. Cuong, T. V.; Pham, V. H.; Tran, Q. T.; Hahn, S. H.; Chung, J. S.; Shin, E. W.; Kim, E. J. Mater. Lett. 2010, 64, 399. 